\title{
Demokratisierung von Technikfolgenabschätzung?
}

\author{
Zum Problem der Verhältnisbestimmung von Öffentlichkeit \\ und wissenschaftlicher Expertise
}

Stefan Böschen, SoTec, RWTH Aachen University, Theaterplatz 14, 52062 Aachen (stefan.boeschen@humtec.rwth-aachen.de) (1) https://orcid.org/0000-0003-0519-5030 Hans-Jörg Sigwart, Institut für Politische Wissenschaft, RWTH Aachen University (sigwart@ipw.rwth-aachen.de) @ https://orcid.org/0000-0002-6151-2945

Technikfolgenabschätzung (TA) öffnet sich gegenwärtig neu für die Beratung demokratischer Öffentlichkeit. Dabei zeigt sich jedoch ein vielschichtiges Positionierungsproblem für TA. Damit TA diese Positionierung transparent vollziehen kann, so unsere These, bedarf es eines demokratietheoretischen Referenzrahmens, in dem die Bezüge zu Öffentlichkeit geklärt werden können. Ein solcher Referenzrahmen kann heuristisch aus den drei Dimensionen politischen Könnens, politischen Wollens und politischen Sollens gebildet werden. Anhand einer Analyse entlang dreier typischer Bezugsformen - Öffentlichkeit als Adressat von TA-Wissen, als Quelle von TA-Wissen und als Interaktionspartner - wird aufgezeigt, dass unter Anwendung eines solchen Referenzrahmes TA ein kritisch-reflexives Verhältnis zu Öffentlichkeit einnehmen und so das Positionierungsproblem produktiv lösen kann.

\section{Democratization of technology assessment?}

The problem of defining the relationship between public sphere and scientific expertise

Technology assessment (TA) is becoming more open to democratic public consultation. This poses a complex problem of self-positioning for TA. We argue that in order for TA to be able to carry out this public self-positioning transparently, a democratic theoretical framework is required which allows clarifying the relationship to the public. Such a frame of reference can be formed from the three dimensions of political ability ("politisches Können"), political willing ("politisches Wollen"), and political obligation ("politisches Sollen"). Based on an analysis along three typical forms - the public as addressee of TA knowledge, as source of TA knowledge, and as interaction partner of TA - we demonstrate how this framework can help TA develop a critical and reflexive relationship to the public and thus productively solve the self-positioning problem.

Keywords: democratization, public sphere, problem-oriented research, critique

This is an article distributed under the terms of the Creative Commons Attribution License CCBY 4.0 (https://creativecommons.org/licenses/by/4.0/)

https://doi.org/10.14512/tatup.29.3.18

Submitted: 17. 06.2020. Peer reviewed. Accepted: 21.10.2020

\section{Einleitung}

Technikfolgenabschätzung (TA) hat sich immer schon als Form der Demokratisierung von politischem Entscheiden verstanden. Ausgehend von parlamentarischer TA (Petermann und Grunwald 2005; Delvenne et al. 2011) öffnete sich TA im Zuge des „Participatory Turn“ (Jasanoff 2003) immer stärker zur Öffentlichkeit, wobei dieser Bezug oftmals instrumentell verengt (Bogner 2010), jedoch im Sinne einer Gesellschaftsberatung (Leggewie 2007) anders denkbar war. Gerade die technischen Möglichkeiten erlauben in der Zwischenzeit eine „Inklusionsexpansion“ (Beetz 2005, S. 20), rücken aber auch einen ,algorithmischen Strukturwandel von Öffentlichkeit“ (Hagen et al. 2017) in den Blick. Möchte TA die Öffnung zur Öffentlichkeit vorantreiben, dann steht sie vor der Aufgabe, ein Verständnis von und eine Relationierung $z u$ Öffentlichkeit zu entwickeln, die den vielschichtigen Anforderungen demokratischer Diskurse gerecht werden und TA als problem-orientierter Forschung eine reflektierte Selbstpositionierung innerhalb dieser Diskurse ermöglichen.

Das Problem der Positionierung wurde lange Zeit durch das Postulat „relativer Distanz“ gelöst: „Ohne relative Distanz kann TA auch gegenüber der Öffentlichkeit keine Korrektivfunktion wahrnehmen." (Gloede 1992, S. 325) Relative Distanz bedeutete, sich als TA durch geeignete Maßnahmen gegenüber den verschiedenen Anspruchspositionen in der politischen Arena als unparteiisch zu positionieren. Eingelöst wurde dies durch das Selbstverständnis, die Folgen des Einsatzes und der Verbreitung bestimmter Technologien abzuschätzen. Diese Positionierung erscheint auf den ersten Blick unverfänglich, sie ist es aber nicht. Denn sie unterschlägt: Folgen werden typischerweise in Relation zu Prozessen gesellschaftlicher Problembearbeitung betrachtet, in denen sich die Formierung der Problemdefinition sowie entsprechender Lösungen öffentlich-politisch vollzieht, so dass die Abschätzung einen normativ gebundenen Akt darstellt. Somit kann relative Distanz nicht allein durch (vermeintlich) objektives Wissen sichergestellt, sondern muss durch die Transparenz normativer Bezüge beim Problemlösen erweitert 
werden. Um dies zu verdeutlichen und analytisch beschreibbar zu machen, so unsere These, bedarf es eines demokratietheoretischen Referenzrahmens, der spezifische Qualitäten demokratischer Diskurse herauszustellen erlaubt.

Unsere Analyse des Selbstpositionierungsproblems von TA geht in drei Schritten vor. Wir skizzieren zunächst drei typische Bezugsformen von TA zu Öffentlichkeit: Öffentlichkeit als Adressat von TA, Öffentlichkeit als Quelle von TA-Wissen sowie Öffentlichkeit als Interaktionspartner. Zweitens entwerfen wir einen Referenzrahmen, um die unterschiedlichen, sich oft scharf voneinander abgrenzenden Akzentsetzungen in der gegenwärtigen Debatte in eine differenzierte Perspektive auf drei Grundfunktionen demokratischer Politik und die ihnen entsprechenden Semantiken öffentlichen Sprechens zu übersetzen: politisches Können, politisches Wollen und politisches Sollen. Schließlich zeigen wir mit Blick auf die drei typischen Bezugsformen von TA zu Öffentlichkeit auf, wie TA ihre Position als politisches Können im Raum der Öffentlichkeit unter Einbeziehung der eigensinnigen Semantiken politischen Sollens und Wollens bestimmen kann und so das Positionierungsproblem besser adressiert (3.).

\section{Bezugsformen zwischen TA und Öffentlichkeit}

Im Sinne einer allgemeinen Definition lässt sich TA als problem-orientierte Forschung bestimmen, die öffentlich-politisch thematisierte Problemlagen aufgreift und Wissen zu deren weiteren Thematisierung sowie Entscheidung bereitstellt. Unter diesem Gesichtspunkt ist es für TA bedeutsam, möglichst genau die öffentlich-politische Thematisierung nachzuvollziehen und in ihrer Analyse zu berücksichtigen. Solange TA auf Einzeltechnologien konzentriert blieb, konnte dieser Bezug mehr oder we-
Wissen ab und damit zugleich auf Allparteilichkeit, um als Beratung nicht in die Nähe ausgesuchter Akteursgruppen zu geraten. Bei dieser Bezugsform stellen sich insbesondere Herausforderungen, die Kommunikationsmittel angemessen zu wählen. TA folgte lange Zeit einer expertenzentrierten Form der Wissenskommunikation und nahm die Kritik am linearen Modell der Kommunikation (Bucchi und Neresini 2008) erst zeitverzögert zur Kenntnis. Öffentlichkeit zu adressieren wird in der gegenwärtig stärker polarisierenden Kommunikation in immer fragmentierteren Arenen deutlich herausfordernder. Denn es bedeutet, dass die Allparteilichkeit nur durch weitergehende Transparenz der eigenen Voraussetzungen gesichert werden kann.

Zweitens ist Öffentlichkeit Quelle von TA-Wissen. In vielen partizipativen Verfahren geht es letztlich darum, dass Laienwissen für den Umgang mit Technologien oder auch das Wertewissen von Bürgerinnen und Bürgern mobilisiert werden soll. Hier hat TA eine Pionierrolle eingenommen und eine Fülle von Methodenwissen erarbeitet. Zugleich blieb eine Asymmetrie in der Wertstellung von Expertenwissen gegenüber Laienwissen wirksam (Bogner 2010). TA nutzt mithin Öffentlichkeit bisher zu wenig als Quelle von Wissen, obgleich dies als problemorientierte Forschung sehr naheliegend wäre. Es steht zu vermuten, dass TA bisher noch nicht über die Instrumente verfügt, um herausfiltern zu können, welches Wissen wofür als nützlich und relevant anzusehen ist - und zwar: transparent und begründet.

Drittens ist Öffentlichkeit Interaktionspartner. Öffentlichkeit ist nicht allein Arena, sondern wird etwa durch zivilgesellschaftliche Akteure und ihre Mobilisierung als „Bewegungsöffentlichkeiten" formiert. Dadurch entstehen mitunter überraschende Relationen. Sprechender Ausdruck dafür ist die Differenz zwischen ,eingeladener" und „,uneingeladener" Partizipation der Zivilgesellschaft (Wehling und Viehöver 2013). TA folgt vielfach

\section{TA kann relative Distanz zur Öffentlichkeit nur sicherstellen, wenn sie sich auch den politischen und normativen Voraussetzungen von Partizipation zuwendet.}

niger ignoriert werden. Mit der Analyse von Prozessen wie etwa der Energiewende jedoch, bei der verschiedene Innovationen Erneuerbarer Energien mit der Exnovation etablierter Technologien (z. B. Kohleverstromung) verzahnt sind, treten die Differenzen bei der Definition zu lösender Probleme sowie der zur Problemlösung jeweils effektiven wie legitimen Strategien sinnfällig vor Augen. Als Bezugsformen von TA zu Öffentlichkeit lassen sich im Wesentlichen die drei folgenden unterscheiden (Rowe und Frewer 2005).

Erstens ist Öffentlichkeit Adressat von TA-Expertise. TA als Forschung und Expertise zielt auf universalisierungsfähiges einem Einladungsmodus und hat dabei Partizipation mitunter zum Labor gemacht (Bogner 2010). Zudem kommen bisher ungeahnte Interaktionspartner ins Spiel, wenn mittels softwarebasierter Desinformationsstrategien Akteure den Verlauf öffentlicher Debatten zu manipulieren trachten (European Commission 2018).

In allen drei genannten Relationen wird deutlich, dass TA relative Distanz in den Bezügen zur Öffentlichkeit nur sicherstellen kann, wenn sie sich neben der instrumentellen Seite der Expertise auch den politischen und normativen Voraussetzungen dieser Expertise zuwendet und hierfür ein Instrumentarium zur 
Selbstreflexion und -positionierung entwickelt. Hierzu stellen wir im Folgenden einen demokratietheoretischen Referenzrahmen vor, der die spezifischen Qualitäten demokratischer Diskurse herauszustellen erlaubt.

\section{Ein demokratietheoretischer Referenzrahmen}

Für die Entfaltung eines solchen demokratietheoretischen Referenzrahmens schlagen wir vor, die heuristische Unterscheidung von politischem Können, politischem Wollen und politischem Sollen als drei zentralen Funktionen demokratischer Diskurse zu nutzen (Beardsworth et al. 2020). Zum einen lässt sich zeigen, wie sich die wissenschaftliche Debatte zur politischen Rolle von TA teils explizit, teils implizit und mit wechselnden Schwerpunkt- und Akzentsetzungen auf diese drei Funktionen bezieht. Zum anderen lässt sich anhand dieser Unterscheidung aufzeigen, dass in öffentlichen Diskursen stets simultan differente politische Grundanliegen adressiert werden. Neben Fragen kompetenter governance auf Grundlage von (technischer) Expertise werden zugleich die fundamentalen demokratischen Belange kollektiver Willensbildung und moralischer Selbstverständigung verhandelt. Diesen Grundanliegen entsprechen drei unterscheidbare Semantiken öffentlichen Sprechens. Mit der Nutzung der genannten Heuristik lassen sich entstandene Verwirrungen innerhalb der öffentlichen Selbstpositionierung von TA entwirren. Um dies zu verdeutlichen, charakterisieren wir im Folgenden knapp die drei Semantiken.

Demokratische Diskurse haben erstens eine Umsetzungsfunktion, nach der es in demokratischer Politik vor allem darum geht, effiziente politische Entscheidungsfindung und gesamtgesellschaftliche Steuerung zu ermöglichen (Mayntz und Scharpf 1995), das heißt wirksame Formen der governance als Mittel
Die auf dieser Grundlage generierten Umsetzungskapazitäten demokratischer Diskurse umfassen verschiedenste Formen von Wissen (Nullmeier 1993; Schuppert 2008), angefangen von Fakteninformationen und technischem Fachwissen bis hin zur Fähigkeit, die Funktionsweise politischer Prozesse und Institutionen zu verstehen sowie zu beurteilen, welche Ziele überhaupt im Bereich des politisch Realisierbaren liegen und welche Kosten und nicht-intendierten Nebenwirkungen ihre Umsetzung impliziert. Die Semantik politischen Könnens konzentriert sich somit auf Sachprobleme, die zwar immer auch Normen umfassen können, diese aber vor allem im Sinne von Koordinationsregeln in Prozessen der Problembearbeitung adressieren. Normativ ist der Semantik politischen Könnens daher die Orientierung an den „Werten“ der Neutralität und Objektivität im Rahmen von ,good governance“ (Czada 2010) eingeschrieben. Es geht dabei um die Fähigkeit, diejenigen objektiven Probleme zu bestimmen, zu verstehen und zu handhaben, die sich unabhängig von den normativen Zielvorgaben kollektiver Willensbildung und moralischer Überzeugungen im Sinne eines kompetenten und verantwortungsvollen Problemlösungshandelns als politische Aufgaben stellen.

Die Verständigung über solche normativen Vorgaben ist aber ebenfalls wesentlicher Bestandteil demokratischer Diskurse. Sie stellen daher zweitens den Versuch dar, über Austausch, Konflikt, aber auch über Zusammenführung unterschiedlicher Meinungen, Interessen und Vorstellungen von den Zielen politischer Gestaltung einen Prozess kollektiver Willensbildung zu ermöglichen (politisches Wollen). In der Semantik politischen Wollens kommt normativ die demokratische Grundidee kollektiver Selbstbestimmung und mit ihr ein Verständnis von politischer Macht nicht nur als ,konstituiert“, sondern auch als „,konstituierend“ zum Ausdruck (Kalyvas 2005, S. 227 ff.). Die Macht der Öffentlichkeit ist hier also als ein Bottom-up-Phänomen zu verstehen, d.h. als ,die Macht freier und gleichberechtigter Bürger als kollektiver Körper“" (Rawls 2003, S. 222).

\section{Der Referenzrahmen zeigt, dass die Rolle von TA traditionell vor allem in der Dimension politischen Könnens gesehen wurde.}

zur Verwirklichung der politischen Selbstgestaltung der Gesellschaft zu generieren und erfolgreich anzuwenden (politisches Können). Eine spezifische Semantik politischen Könnens in diesem Sinne entfalten demokratische Diskurse in erster Linie im Medium konstituierter Macht, d. h. in den institutionalisierten Formen demokratischer Politik. Diese umfassen neben den klassischen repräsentativen, Regierungs- und gesellschaftlichen Vermittlungsinstitutionen auch vielfältige hybride Formen von governance, an denen auch Netzwerke aus Nichtregierungsorganisationen, Wirtschaftsunternehmen und einzelnen Bürgern als Akteure beteiligt sind.
Realiter besteht dieser „,kollektive Körper“ bzw. die Öffentlichkeit als Ganzes zwar immer aus einer Vielzahl oft widersprüchlicher Positionen, und Prozesse der kollektiven Willensbildung vollziehen sich vor allem in der Form von andauernden und nicht vollständig auflösbaren Konflikten zwischen ihnen. Aber in einem solchen pluralistisch-agonalen Sinne verstanden ist die Semantik politischen Wollens dennoch ein reales Element demokratischer Politik, das sich in konkreten Formen des ,,practical enactment in public life“ (White und Ypi 2017, S. 444) artikuliert und reale Effekte auf die Agenda demokratischer Prozesse hat. Das politische Wollen der Öffentlichkeit in diesem 
Sinne artikuliert sich faktisch etwa in grundlegenden Neujustierungen öffentlicher Selbstverständigungsdebatten, die sowohl die Form von fundamentalen „Ergänzungen“ als auch von (zumindest partiellen) „Unterbrechungen“ bisheriger öffentlicher Debatten und entsprechender Entwicklungstendenzen annehmen können (Wenman 2013).

Im demokratischen Diskurs über die normativen Grundkoordinaten des politischen Prozesses geht es aber nicht ausschließlich um die Organisierung kollektiver Willensbildungsprozesse, sondern - drittens - auch um den Versuch, Kategorien moralischer Orientierung zu generieren und Grundprinzipien der öffentlich-politischen Moral zu artikulieren, die z. B. schützenswerte Grundrechte als unverzichtbare Voraussetzungen der legitimen Anwendung politischer Gestaltungsmacht bestimmen (politisches Sollen). Dieser moralische Charakter öffentlicher Diskurse drückt sich vor allem darin aus, dass in ihnen immer auch die Frage nach den grundlegenden Einschränkungen mitverhandelt wird, denen politisches Handeln unterworfen sein soll (Rawls 2002, S. 27 f.). Die entsprechende Semantik politischen Sprechens folgt einer im Anspruch objektiven und universalen, aber in erster Linie negativen Logik der Bestimmung der moralischen Grenzen nicht nur des politisch „Machbaren“, sondern auch der legitimen „Willkür“ des demokratischen Souveräns (ebd., S. 31).

In der Semantik politischen Sollens spiegelt sich somit die genuin liberale Idee, dass jede politische Regierung, auch eine solche, die sich auf das demokratische Prinzip der Volkssouveränität beruft, d.h. dass sowohl die Erfordernisse wirksamer governance als auch die Prozesse kollektiver Willensbildung der moralischen Beschränkung bedürfen. Der Hinweis auf den souveränen Willen des Volkes kann alleine weder eine normativ zufriedenstellende Vermittlung zwischen kollektiver und individueller Autonomie garantieren (Habermas 1992, S. 112 ff.), noch den Status von Individuen als moralische Subjekte sicherstellen (Habermas 2002). Im Unterschied zur voluntaristischen Semantik kollektiver Willensbildung artikuliert sich politisches Sollen daher in einer Semantik der grundlegenden moralischen Pflichten, an deren Erfüllung jede demokratische Gemeinschaft normativ gebunden sein soll.

Wenden wir den Blick auf TA und betrachten ihre Entwicklung im Lichte des hier skizzierten Referenzrahmens, dann zeigt sich, dass die Rolle von TA traditionell zunächst vor allem in der Dimension politischen Könnens gesehen wurde. Gegenwärtig deutet die kontrovers geführte Diskussion zur Frage der normativen Implikationen von TA, in deren Verlauf der Anspruch von TA auf Neutralität zugunsten eines stärker ethisch-moralischen (Grunwald 2017, S. 317 ff.) oder eines dezidiert politischen Verständnisses (Delvenne und Parotte 2019) reflektiert wird, auf eine Öffnung in Richtung der Dimensionen politischen Wollens und Sollens hin. Die in der Debatte vorherrschende Positionierung dieser verschiedenen Verständnisse von TA als unvereinbare Alternativen wird jedoch dem komplexen Problem der politischen Selbstpositionierung von TA gerade nicht gerecht. Unsere heuristische Unterscheidung legt stattdessen nahe, dass etwa die Argumente von Grunwald einerseits und Delvenne andererseits (Delvenne et al. 2019) nur aufgrund einer ungenügenden Differenzierung zwischen politischem Können, politischem Sollen und politischem Wollen als sich ausschließende Alternativen erscheinen.

\section{Das Positionierungsproblem von TA revisited}

Mit Blick auf unseren demokratietheoretischen Referenzrahmen muss die Frage der Selbstpositionierung von TA somit als das Problem einer differenzierenden Aufgabenbestimmung beschrieben werden, welche die unterschiedlichen, teils in einer ambivalenten Beziehung zueinander stehenden Anforderungen und Semantiken öffentlicher Kommunikation berücksichtigt. Wie sich eine solche Positionierung im Triangle aus politischem Können, politischem Wollen und politischem Sollen situativ vollziehen könnte, soll nun abschließend hinsichtlich der verschiedenen Bezugsformen von TA zu Öffentlichkeit knapp skizziert werden.

Öffentlichkeit als Adressat von TA-Wissen. So lange sich die Herausforderung auf die Kommunikation wissenschaftsbasierter Expertise bezog, war die Aufgabe für TA relativ einfach, da sie sich mehr oder weniger unhinterfragt auf politisches Können fokussieren konnte. Allerdings zeigt insbesondere die Debatte um wissenschaftliches Nichtwissen (Proctor und Schiebinger 2008; Böschen et al. 2010) neue Reflexionserfordernisse auf. Denn genau besehen verweist Nichtwissen neben der Einsicht in die Grenzen politischen Könnens auf die Notwendigkeit, im Angesicht dieser Grenzen politische Festlegungen über den weiteren Umgang mit Nichtwissen zu treffen. Welche Vorsorgestrategien sollen getroffen werden, welche nicht? Welche Schäden durch unerkanntes Nichtwissen sind hinnehmbar, welche hingegen nicht? Dies sind genuin Fragen politischen Wollens und Sollens. Das Problem des Nichtwissens ist inmitten des Triangels von politischem Können, Wollen und Sollen platziert. Insofern bedarf es einer reflektierten Situierung von TA-Wissen im Spannungsfeld der durch Nichtwissen gezogenen Grenzen, mitlaufenden Strategien und politischen Interessen. Die offensive Äußerung von Zweifel kann Ausdruck eines politischen Wollens sein, wie sich in der Klimawandelskepsis deutlich zeigt (Oreskes und Conway 2010). Vor diesem Hintergrund macht der Referenzrahmen zwei wichtige Angebote für TA. Erstens ermöglicht es dieser Rahmen, die Nutzung und das Wirksam-machen von Nichtwissen in öffentlichen Debatten zu rekonstruieren, indem er in den jeweiligen Artikulationen von Nichtwissen die drei öffentlichen Semantiken und damit die genuin politischen Qualitäten dieser Wissensform abschichtet. Zweitens hilft er dabei, die wissenskommunikative Herausforderung zu lösen, genau diese Merkmale transparent zu machen.

Öffentlichkeit als Quelle von TA-Wissen. Gerade bei etablierten Verfahren der Partizipation verdeutlicht der Blick mittels der Semantiken eine entscheidende Schieflage. Denn diese 
sind primär in der Logik politischen Könnens und Sollens verhaftet. In der Konsequenz werden Akteure am politischen Können zwar beteiligt und dabei mitunter auch moralische Fragen politischen Sollens adressiert, dies jedoch bezeichnenderweise unter Nichtberücksichtigung der spezifischen Semantik politischen Wollens und ihrer Implikationen. Diese Selektivität verdankt sich dem Umstand, dass TA mit der Grundprämisse eines argumentativ-rationalen Räsonierens operierend ein konzeptionelles Ungleichgewicht zu Gunsten politischen Könnens institutionalisiert hat (Hennen 2012). Dies erschwert auch das Verständnis aktuell virulenter Probleme. So lässt sich etwa das Problem fragmentierter Öffentlichkeiten in seinen Implikationen für TA adäquat nur unter Berücksichtigung der besonderen $\mathrm{Se}$ mantik demokratischer Willensbildungsprozesse adressieren. $\mathrm{Si}$ cherlich kann Öffentlichkeit unter den Bedingungen hoher Fragmentierung weiterhin als Quelle von TA-Wissen dienen. Jedoch stellt die Fragmentierung ein zentrales Problem dar, sobald es um die Einbeziehung der Semantik öffentlicher Willensbildungsprozesse, d. h. des „Wollens“ der Öffentlichkeit und entsprechender hegemonialer Deutungen (Laclau und Mouffe 1991, S. 100 ff.) oder anders ausgedrückt sobald es um Öffentlichkeit als eines in welcher Weise auch immer integrierten Gesamtzusammenhangs geht (Dewey 1996, S. 134 ff.). Der Referenzrahmen eröffnet hier also für TA die Möglichkeit, trotz Auflösung klarer politischer Relevanz- und Orientierungsrahmen für die Problemorientierung, Positionierungsweisen zu identifizie- ziell in einer Wollens-Semantik von immer nur auf „politischer“ Ebene zu entscheidenden „Wertkonflikten“ aufgelöst werden (Weydner-Volkmann 2019). Mit dem Referenzrahmen kann TA gegenüber der Öffentlichkeit als Interaktionspartner transparenter der Aufgabe nachkommen, aktiv zur Klarheit und Aufrechterhaltung entsprechender Differenzierungen und einer entsprechenden Praxis öffentlicher Selbstverständigung beizutragen.

\section{Fazit}

Die hier skizzierte Anwendung unseres Referenzrahmens zeigt, wie etablierte Formen von TA reflektiert und anders positioniert werden können und inwiefern die Differenzierung unterschiedlicher Semantiken öffentlichen Sprechens zentrale Aspekte des Positionierungsproblems von TA in Öffentlichkeit zu identifizieren hilft. So zeigt sich zum einen, dass eine reflexive Praxis der Selbstverortung von TA insbesondere gegenüber der Logik politischen Wollens nicht mehr umstandslos im alten Postulat einer mehr oder weniger fixierten ,relativen Distanz" vollzogen werden kann. Denn beim politischen Wollen ist der Adressat die Gesellschaft bzw. die Öffentlichkeit, die TA nicht als ein Gegenüber verstehen kann. Hier muss TA ihrem öffentlichen Charakter vielmehr im Sinne eigenen Beteiligt-Seins gerecht werden. Ein genuin kritischer Anspruch von TA gegenüber dem politi-

\section{Der Referenzrahmen eröffnet für TA die Möglichkeit, Problemlösungsperspektiven genauer zu erfassen.}

ren und so das Panorama der Problemlösungsperspektiven genauer zu erfassen. Jedoch ist nicht zu übersehen, wie aufwändig diese Aufgabe angesichts der Fragmentierung von Öffentlichkeit mitunter sein kann.

Öffentlichkeit als Interaktionspartner. Insbesondere Verfahren der Constructive TA haben prägnant diesen Aspekt des Öffentlichkeitsbezugs in den Blick gerückt und praktiziert. Gerade dem politischen Wollen wurde so Spielraum gegeben. Jedoch wurde hierbei, paradoxerweise, der Aspekt politischen Könnens in den Hintergrund gerückt und systematisch ausgeblendet, dass etwa der Umstand des Von-vornherein-Einbeziehens nicht schon automatisch die Vermeidung von negativen Folgen bedeutet. Einer pauschalen Kritik an CTA, welche den Umstand gemeinsamer Entwicklungsarbeit als Ausschluss einer kritischen Perspektive deutet, würden wir zwar entgegnen, dass dieses Argument das politische Wollen nicht ernst nimmt und verkennt, dass bereits jeder Versuch einer öffentlichen Problembeschreibung unweigerlich die Logik kollektiver Willensbildungsprozesse mit involviert (Delvenne und Parotte 2019). Andererseits dürfen die sehr unterschiedlichen normativen Logiken politischen Sollens und politischen Wollens nicht vermischt und tenden- schen Wollen der Öffentlichkeit kann folgerichtig nicht schlicht mit Verweis auf wissenschaftliche Distanz, Neutralität und Objektivität begründet werden, sondern ist im Aufweis eines spezifisch kritischen Modus des Beteiligt-Seins einzulösen. Zum anderen zeigt sich aber auch, dass dieser Befund die Frage nach der öffentlichen Rolle wissenschaftlicher Neutralität und Objektivität keineswegs einfach obsolet macht. Die Differenzierung politischen Könnens und Sollens als eigenständige und wesentliche Funktionen von Öffentlichkeit kann vielmehr gerade helfen, den öffentlichen Ort und die demokratische Rolle des für TA weiterhin konstitutiven kritischen Anspruchs auf ,relative Distanz“ in neuer Klarheit zu bestimmen.

\footnotetext{
Literatur

Beardsworth, Richard; Behr, Hartmut; Sigwart, Hans-Jörg (2020): Anti-democratic distortions and the future of liberal democracy. Negotiating the national-

international tension. Unpublished working paper in progress.

Beetz, Michael (2005): Die Rationalität der Öffentlichkeit. Konstanz: UVK.

Bogner, Alexander (2010): Partizipation als Laborexperiment. Paradoxien der Laiendeliberation in Technikfragen. In: Zeitschrift für Soziologie 39 (2), S. 87-105.
} 
Böschen, Stefan; Kastenhofer, Karen; Rust, Ina; Soentgen, Jens; Wehling, Peter (2010): Scientific nonknowledge and its political dynamics. The cases of agri-biotechnology and mobile phoning. In: Science, Technology \& Human Values 35 (6), S. 783-811. DOI: 10.1177/0162243909357911.

Bucchi, Massimiano; Neresini, Federico (2008): Science and public participation. In: Edward Hackett, Olga Amsterdamska, Michael Lynch and Judy Wajcman (Hg.): The handbook of science and technology studies. Cambridge (Massachusetts): MIT-Press, S. 449-472.

Czada, Roland (2010): Good Governance als Leitkkonzept für Regierungshandeln. Grundlagen, Anwendungen, Kritik. In: Arthur Benz und Nicolai Dose (Hg.): Governance - Regieren in komplexen Regelsystemen. Eine Einführung. Wiesbaden: vS Verlag für Sozialwissenschaften, S. 201-224.

Delvenne, Pierre; Grunwald, Armin; Nierling, Linda; Torgersen, Helge (2019): Interview. Balancing engagement and neutrality in technology assessment. In: TATuP - Zeitschrift für Technikfolgenabschätzung in Theorie und Praxis 28 (1), S.71-74. DOI: 10.14512/tatup.28.1.71

Delvenne, Pierre; Parotte, Celine (2019): Breaking the myth of neutrality. Technology assessment has politics, technology assessment as politics. In: Technological Forecasting and Social Change 139, S. 64-72. DOI: 10.1016/ j.techfore.2018.06.026

Delvenne, Pierre; Fallon, Christine; Brunet, Sébastien (2011): Parliamentary technology assessment institutions as indications of reflexive modernization. In: Technology in Society 33 (1-2), S. 36-43. D0I: 10.1016/j.techsoc.2011.03.004

Dewey, John (1996): Die Öffentlichkeit und ihre Probleme. Darmstadt: Wissenschaftliche Buchgesellschaft.

European Commission (2018): A multi-dimensional approach to disinformation. Report of the independent high level group on fake news and online disinformation. Brussels: Directorate-General for Communication Networks, Content and Technology.

Gloede, Fritz (1992): Rationalisierung oder reflexive Verwissenschaftlichung? Zur Debatte um die Funktionen von Technikfolgen-Abschätzung für Technikpolitik. In: Thomas Petermann (Hg.): Technikfolgen-Abschätzung als Technikforschung und Politikberatung. Frankfurt: Campus, S. 299-328.

Grunwald, Armin (2017): Technik und Politikberatung. Philosophische Perspektiven. Frankfurt am Main: Suhrkamp.

Habermas, Jürgen (1992): Faktizität und Geltung. Beiträge zur Diskurstheorie des Rechts und des demokratischen Rechtsstaats. Frankfurt am Main: Suhrkamp. Habermas, Jürgen (2002): Die Zukunft der menschlichen Natur. Auf dem Weg zu einer liberalen Eugenik. Frankfurt am Main: Suhrkamp.

Hagen, Lutz; Wieland, Mareike; In der Au, Anne-Marie (2017): Algorithmischer Strukturwandel der Öffentlichkeit. In: MedienJournal 41 (2), S. $127-43$.

Hennen, Leonhard (2012): Why do we still need participatory Technology Assessment? In: Poiesis \& Praxis 9 (1-2), S. 27-41. DOI: 10.1007/s10202-012-0122-5.

Jasanoff, Sheila (2003): Technologies of humility. Citizen participation in governing science. In: Minerva 41 (3), S. 233-244.

Kalyvas, Andreas (2005): Popular sovereignty, democracy, and the constituent power. In: Constellations 12 (2), S. 223-244. DOI: 10.1111/j.13510487.2005.00413.x.

Laclau, Ernesto; Mouffe, Chantal (1991): Hegemonie und radikale Demokratie. Zur Dekonstruktion des Marxismus, Wien: Passagen.

Leggewie, Claus (Hg.) (2007): Von der Politik- zur Gesellschaftsberatung. Frankfurt am Main: Campus.

Mayntz, Renate; Scharpf, Fritz (Hg.) (1995): Gesellschaftliche Selbstregelung und politische Steuerung, Frankfurt am Main: Campus.
Nullmeier, Frank (1993): Wissen und Policy-Forschung. Wissenspolitologie und rhetorisch-dialektisches Handlungsmodell. In: Adrienne Héritier (Hg.): Policy-Analyse. Kritik und Neuorientierung. Opladen: Westdeutscher Verlag, S. 175-196.

Oreskes, Naomi; Conway, Erik (2010): Merchants of doubt. New York: Bloomsbury Press.

Petermann, Thomas; Grunwald, Armin (Hg.) (2005): Technikfolgen-Abschätzung für den Deutschen Bundestag. Das TAB - Erfahrungen und Perspektiven wissenschaftlicher Politikberatung. Berlin: edition sigma.

Proctor, Robert; Schiebinger, Londa (Hg.) (2008): Agnotology. The making and unmaking of ignorance. Stanford: Stanford University Press.

Rawls, John (2002): Das Recht der Völker. Berlin: Walter de Gruyter.

Rawls, John (2003): Politischer Liberalismus. Frankfurt am Main: Suhrkamp.

Rowe, Gene; Frewer, Lynn (2005): A typology of public engagement mechanisms. In: Science, Technology \& Human Values 30 (2), S. 251-290. D0I: 10.1177/ 0162243904271724.

Schuppert, Gunnar (2008): Governance durch Wissen. Überlegungen zum Verhältnis von Macht und Wissen aus governancetheoretischer Perspektive. In: Gunnar Schuppert und Andreas Voßkuhle (Hg.): Governance von und durch Wissen. Baden-Baden: Nomos, S. 259-304.

Wehling, Peter; Viehöver, Willy (2013): ,Uneingeladene' Partizipation der Zivilgesellschaft. Ein kreatives Element der Governance von Wissenschaft. In: Edgar Grande, Dorothea Jansen, Otfried Jarren, Arie Rip, Uwe Schimank und Peter Weingart (Hg.): Neue Governance der Wissenschaft. Bielefeld: transcript, S. 213-234.

Wenman, Mark (2013): Agonistic democracy. Constituent power in the era of globalization. Cambridge: Cambridge University Press.

Weydner-Volkmann, Sebastian (2019): Ethische Technikfolgenabschätzung als Kartographie situativer Wertungskonflikte. In: TATuP - Zeitschrift für Technikfolgenabschätzung in Theorie und Praxis 28 (1), S. 39-44. D0I: 10.14512/ tatup.28.1.39

White, Jonathan; Ypi, Lea (2017): The politics of peoplehood. In: Political Theory 45 (4), S. 439-465. DOI: 10.1177/0090591715608899.

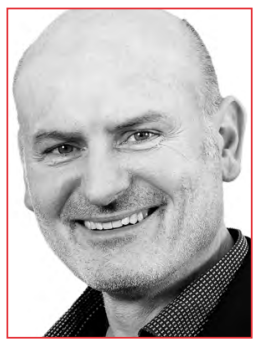

\section{PROF.DR.STEFAN BÖSCHEN}

leitet den Lehrstuhl „Technik und Gesellschaft" am Human Technology Center (HumTec) der RWTH Aachen University. Er ist Chemie-Ingenieur und Soziologe mit Schwerpunkten in der Wissenschaftsund Technikforschung sowie Technikfolgenabschätzung

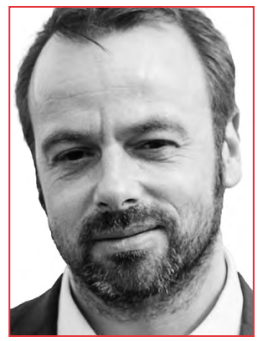

\section{PROF.DR. HANS-JÖRG SIGWART}

leitet den Lehrstuhl für Politische Theorie und Ideengeschichte am Institut für Politische Wissenschaft der RWTH Aachen University. Seine Schwerpunkte liegen in der zeitgenössischen politischen Theorie und Demokratietheorie sowie in der politischen Ideengeschichte des 20. Jahrhunderts. 